\title{
Magneto-Convection: Structure and Dynamics
}

\author{
Robert F. Stein ${ }^{1}$ and Åke Nordlund ${ }^{2}$ \\ ${ }^{1}$ Department of Physics and Astronomy, Michigan State University, East Lansing, MI 48824, \\ USA, email: stein@pa.msu.edu \\ ${ }^{2}$ NBIfAFG, Copenhagen University, Copenhagen, DK, email: aake@astro.ku.dk
}

\begin{abstract}
We present results from realistic, high resolution, simulations of solar magnetoconvection. Simulations were run with both a mean vertical and a mean horizontal field. The magnetic field is quickly swept out of the granules and meso-granules and concentrated in the intergranular lanes.
\end{abstract}

\section{Introduction}

Observations with the $1 \mathrm{~m}$ Swedish Solar Telescope are showing the solar surface at resolutions down to $70 \mathrm{~km}$. SolarB and the Advanced Technology Solar Telescope will produce even more new information on the structure of the solar surface and its magnetic field configurations. Local helioseismology has revealed the dynamic and thermal structure of the near surface layers of the Sun on scales of supergranulation and larger. To interpret and understand these new observations it behooves us to simulate the behavior of solar magneto-convection and study its properties and observable manifestations.

Solar surface dynamics is driven by turbulent convection, magnetic fields and the escape of radiation. Convection transports energy upward through the outer third of the sun and radiation carries it away to space. Convective motions transport angular momentum and produce differential rotation and the subsurface shear layer (Brummell et al. 1998, Miesch et al. 2000a). Convection builds magnetic fields by dynamo action and transports it downward (Nordlund et al. 1992, Tobias et al. 2001, Emonet and Cattaneo 2001). Magnetic flux which emerges through the surface is shuffled around by the convective motions (Berger and Title 1996, Lin and Rimmele 1999). This stresses the field whose subsequent relaxation heats the chromosphere and corona and controls their structure (Galsgaard and Nordlund 1996). Convective motions generate the waves that produce the enhanced emission observed from the chromosphere (Carlsson and Stein 1997, Skartlien et al. 2000). Convection excites the p-mode oscillations and modifies their eigenfrequencies (Stein et al. 2004). Magnetic fields, oscillations and rotational shearing all alter convection itself (Brummell et al. 1998, Steiner et al. 1998). The interaction of these various processes controls the flux of radiation received by the Earth.

In this paper we present some results from realistic, high resolution, simulations of solar surface magneto-convection.

\section{Method}

Our simulations solve the conservation equations for mass, momentum and internal energy per unit mass, together with the induction equation for the vector potential. To determine the radiative heating and cooling in the energy conservation equation, we solve the radiative transfer equation. We assume LTE and a non-gray opacity grouped into 4 bins according to strength. We perform a formal solution of the Feautrier equation along a vertical and four straight, slanted, rays (at four azimuthal angles which are 

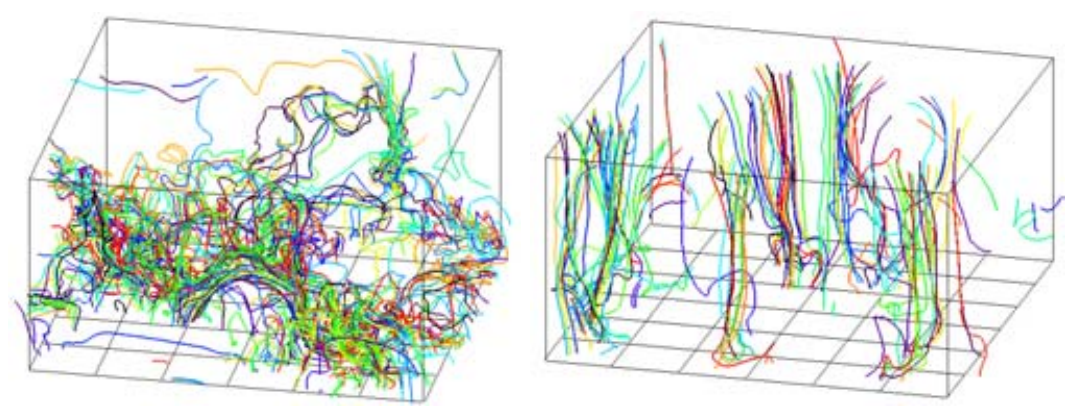

Figure 1. Magnetic field lines for case of advection of horizontal field by inflows at the bottom (note blue horizontal field line at lower left) (left) and case of vertical field at the bottom boundary (right). The magnetic field is swept out of the mesogranule centers to their boundaries and concentrated into kilogauss strength. Note the rising loop in the lower center. There is a "flux tube" extending nearly vertically through the surface in the upper right.

rotated $15 \mathrm{deg}$. every time step). We use a tabular equation of state, which includes LTE ionization and excitation of hydrogen, helium and other abundant atoms as well as molecular hydrogen. The computational domain extends from the temperature minimum at the top of the photosphere to a depth of $2.5 \mathrm{Mm}$ below the mean $\tau_{\text {cont }}=1$ surface. The horizontal extent is $6 \times 6 \mathrm{Mm}$. The horizontal resolution is $25 \mathrm{~km}$ and the vertical resolution $15 \mathrm{~km}$ at the surface increasing to $35 \mathrm{~km}$ at the bottom of the domain. The side boundaries are periodic and the top and bottom boundaries are transmitting. The entropy of inflowing material at the bottom boundary is set so as to closely match the solar luminosity. The magnetic structure depends on the magnetic boundary conditions. We have simulated cases with two different bottom boundary conditions: (1) Inflowing plasma at the bottom advects in horizontal magnetic field with strength about 50G. This models rising weak flux. (2) Magnetic field vertical at the bottom boundary with an average strength of $250 \mathrm{G}$. This models plage and quiet Sun regions. The top boundary condition is that the field tends towards a potential configuration.

\section{Magneto-Convection}

\subsection{Magnetic Field Lines}

Whatever the magnetic field boundary conditions, the field is quickly swept into the intergranular lanes and then to the underlying mesogranule downflow lanes. Magnetic field lines for the case of horizontal field advected in by upflows at mesogranule centers are illustrated in Fig. 1 (left). A fieldline being advected into the computational domain in the mesogranular upflow is visible in the lower left. A rising flux loop is located in the lower center. A nearly vertical "flux tube" is present in the upper right. The field lines are extremely tangled in the mesogranular downflows and in the "flux loop" below the surface, and they connect over long distances. Such "flux tubes" typically form when a loop emerges through the surface and opens up into the overlying atmosphere. One can see from this figure that the concept of "flux tube" is generally not a useful description of magnetic fields near the solar surface.

Magnetic field lines for the case of vertical field are illustrated in Fig. 1 (right). Again the magnetic field is concentrated into the downdrafts, leaving most of the volume with weak field. Close to the surface, where $\beta<1$, the field lines are generally regular, while in the high $\beta$ layers close to the bottom the convection twists the field significantly.

\subsection{Flux Emergence and Disappearance}

In the calculation where horizontal field is advected in at the bottom, instances of both flux loop emergence and disappearance occur. An example both is shown in Fig. 2. 


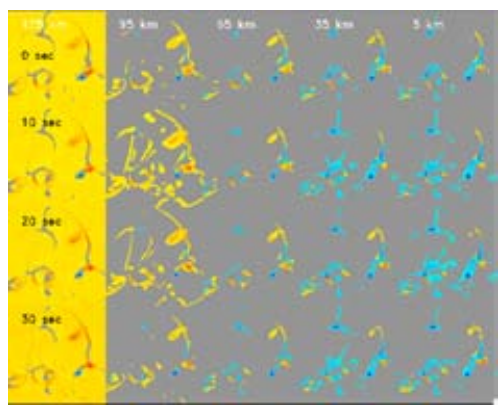

Figure 2. Horizontal slices showing the magnetic field strength at 10 sec intervals at heights from 5 to $125 \mathrm{~km}$ above the mean visible surface. Red and yellow are one polarity, blue is the opposite polarity. Note how the bipole at the right separates in time and is more separated at smaller heights, because this loop is emerging. Note how the bipole at the left is initially visible at all heights, but remains visible only at smaller heights at later times, because this loop is submerging.
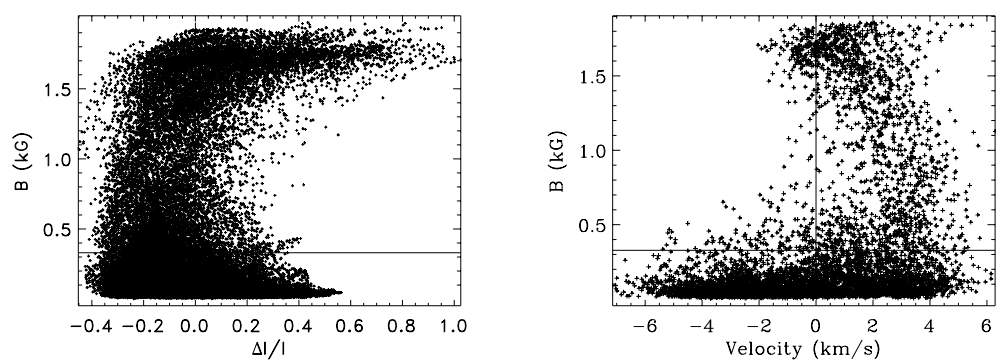

Figure 3. Correlation of magnetic field strength at the mean visible surface with G-band intensity (left) and with vertical velocity (right). G-band bright points correspond to strong magnetic fields, but most strong field locations actually have average G-band brightness and some intense magnetic fields, in wide flux concentrations, appear dark in the G-band. Intermediate strength magnetic concentrations appear dark in the G-band. Magnetic fields are swept into downflow lanes (positive vertical velocity). However, some weak fields $(<400 \mathrm{G}$ ) occur in upflows and some very strong fields occur in weak upflows (negative vertical velocity).

Vertical columns show different heights above the mean visible surface in $30 \mathrm{~km}$ intervals. Horizontal rows show different times at $10 \mathrm{sec}$. intervals. In the lower right of each snapshot is an emerging flux loop whose foot points are more widely separated at lower altitudes and at later times. At the lower left is a magnetic loop that disappears from the surface by submerging. At each time the loop is more widely separated at lower heights. With increasing time the legs of the loop come together and disappear from the larger heights.

\subsection{G-Band}

G-band bright points are often used as a proxy for strong magnetic fields. Fig. 3 illustrates that the very brightest G-band locations do indeed always correspond to strong fields. However, strong fields have a continuous distribution of G-band intensities from brightest to nearly darkest, with most strong fields having average G-band intensity. As has been known for some time, Berger and Title 1996, a significant number of strong field locations are dark in the G-band. These are wide flux concentrations where heating by horizontal radiation transport is not as significant. Intermediate strength fields mostly appear dark in the G-band. We have made one calculation without any horizontal radiative energy transport. In that case there are no G-band bright points. This confirms the 

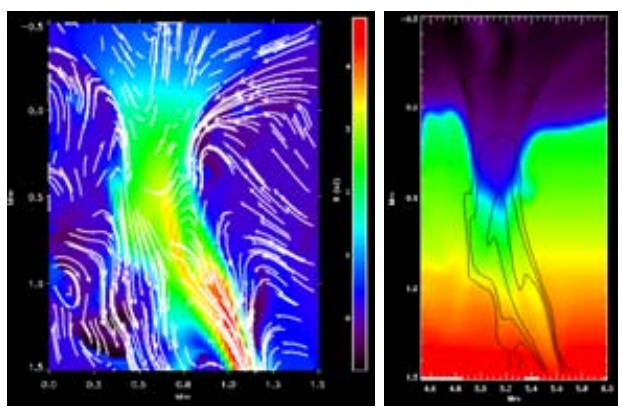

Figure 4. Image of magnetic field strength with superimposed fluid velocity arrows (left) and image of temperature with superimposed magnetic field strength contours at 1, 2, $3 \mathrm{kG}$ (right), in a vertical slice across a magnetic flux concentration.

ideas of Spruit 1977 that heating by hot walls is responsible for small flux concentrations appearing bright.

Diverging convective upflows sweep the magnetic field into the downflows. Generally, the stronger the field the smaller the vertical velocity. However, at the mean visible surface, some very strong field locations are in slow upflows. Weak fields $(<500 G)$ occur over the entire range of upflows and downflows (Fig. 3).

\subsection{Magnetic Concentrations}

Finally, we consider a vertical slice through a magnetic flux concentration. Fig. 4 (left) shows a snapshot of fluid velocities superimposed on an image of magnetic field strength. Near the surface, the granular upflows turn over into very narrow downflow sheaths on the edges of the flux concentration. In the photosphere there is significant upflow in the center of the spreading flux concentration with downflows near its edges. Below $1 \mathrm{Mm}$ there is a large downflow inside the flux concentration, probably representing the continuing evacuation of the flux concentration. The inhibition of convection in the flux concentration, together with its evacuation makes the concentration cooler than its surroundings at all levels below the mean visible surface (Fig. 4 right). Note also the especially cool layers on either side of the magnetic concentration at the visible surface and above. These are possibly due to adiabatic expansion of the plasma in this region and is a very robust feature of our simulated flux concentrations. There is also a cool central core in the photospheric flux concentration due to adiabatic cooling in the diverging upflow.

\section{References}

Berger, T. E. and Title, A. M.: 1996, ApJ 463, 365

Brummell, N. H., Hurlburt, N. E., and Toomre, J.: 1998, ApJ 493, 955

Carlsson, M. and Stein, R. F.: 1997, ApJ 481, 500

Emonet, T. and Cattaneo, F.: 2001, ApJ 560, L197-L200

Galsgaard, K. and Nordlund, Å.: 1996, Journal of Geophysical Research 101(A6), 13445-13460

Lin, H. and Rimmele, T.: 1999, ApJ 514, 448-455

Miesch, M. S., Elliott, J. R., Toomre, J., Clune, T. L., Glatzmaier, G. A., and Gilman, P. A.: 2000a, textitApJ 532, 593-615

Nordlund, Å., Brandenburg, A., Jennings, R. L., Rieutord, M., Roukolainen, J., Stein, R. F., and Tuominen, I.: 1992, ApJ 392, 647-652

Skartlien, R., Stein, R. F., and Nordlund, Å.: 2000, ApJ 541, 468-488

Spruit, H. C. 1976, Sol. Phys., 55, 3

Stein, R. F., Georgobiani, D., Trampedach, R., Ludwig, H.-G., Nordlund, A.: 2004, Sol. Phys. 220, 229

Steiner, O., Grossmann-Doerth, U., Knoelker, M., and Schuessler, M.: 1998, ApJ 495, 468

Tobias, S. M., Brummell, N. H., Clune, T. L., and Toomre, J.: 2001, ApJ 549, 1183-1203 